\section{ECCOMAS}

\section{Proceedia}

$8^{\text {th }}$ ECCOMAS Thematic Conference on Computational Methods in Structural Dynamics and Earthquake Engineering

M. Papadrakakis, M. Fragiadakis (eds.)

\title{
THE TRACKING OF MODAL PARAMETERS FOR A REINFORCED CONCRETE BUILDING DURING LOW-MEDIUM INTENSITY EARTHQUAKES
}

\author{
Davide Arezzo ${ }^{1}$, Vanni Nicoletti ${ }^{1}$, Sandro Carbonari ${ }^{1}$, and Fabrizio Gara ${ }^{1}$ \\ ${ }^{1}$ Dept. ICEA, Università Politecnica delle Marche \\ Via Brecce Bianche, 60131, Ancona, Italy \\ e-mail: d.arezzo@pm.univpm.it, v.nicoletti@pm.univpm.it, s.carbonari@univpm.it, f.gara@univpm.it
}

\begin{abstract}
This paper presents the results of the dynamic monitoring carried out on a school building in central Italy during the seismic sequence following the first main shock of the 2016 Central Italy earthquake. The building is located in the historical centre of Camerino and consists of a reinforced concrete frame structure with masonry infill walls. The school, dating back to the 60 s, underwent seismic retrofit in 2013 through the construction of 2 dissipative towers, a recent patented system for seismic protection of buildings.

In August 2016 a dynamic monitoring system was installed on the building, positioning an array of accelerometers both on the top two floors of the structure and on the foundation level; this made it possible to record the building response to the aftershocks that occurred during the monitoring period, and the corresponding seismic input. The dynamic characteristics of the structure during the monitoring period are identified starting from the response of the structure subjected to the seismic swarm following the main event, and to the environmental vibrations between two subsequent events.

Although during the monitoring days the building did not suffer any damage, the response of the structure proved to be nonlinear and strongly dependent on the amplitude of the accelerations to which it was subjected; in this work, a procedure to linearize the structural response and carry out the dynamic identification in terms of modal parameters starting from the nonstationary response of the structure is proposed.
\end{abstract}

Keywords: Structural Health Monitoring; Infilled RC frame building; Ambient vibrations; Seismic monitoring; Earthquake swarm; Dynamic system identification; Time-varying systems. 


\section{INTRODUCTION}

There has been a progressive increase in attention in recent years to the usefulness and advantages of permanent monitoring systems in the field of civil engineering. Recording the dynamic response of structures during seismic events can ensure significant benefits: on one hand, it provides useful information for damage detection and post-earthquake emergency management and, on the other hand, it can allow the improvement of the seismic risk assessment through the decrease of uncertainties relevant to both the hazard estimation and structural vulnerability. The reduction of uncertainties relevant to the structural vulnerability can derive from the validation of structural models based on the registration of the structural response subjected to low-medium intensity events while the reduction of uncertainties relevant to the hazard requires the monitoring of the soil-foundation system through which the validation of soil and source models can be attempted.

With respect to low-medium intensity earthquakes, an interesting aspect that emerges from the monitoring is the well-known variability of the dynamic properties of structures during shaking in absence of damage producing a "wandering" of the modal parameters of the construction that can be attributed to minor nonlinearities of the response [1] such as the light cracking of infills or frictions relevant to the interactions between structural and non-structural components. The latter issue, in addition to the well-known dependence of structural frequencies on the environmental conditions [2, 3, 4], makes the identification of structural damage from records obtained from continuous monitoring systems particularly problematic. In the literature it is possible to find several works that address the problem with reference to historical masonry buildings $[5,6,7]$, but a limited number of works refer to reinforced concrete structures.

With the aim of providing a contribution in this research area, this paper presents an approach for tracking the fundamental frequencies of monitored buildings accounting for their nonlinear response, triggered by low-medium intensity earthquakes. The procedure is applied to a record extracted from the monitoring of a school building, the Liceo Varano located in Camerino in the province of Macerata (Italy), during the seismic swarm following the earthquake in central Italy in August 2016.

\section{DESCRIPTION OF THE BUILDING}

The building at hand dates back to the 60's and was born from the decision to enlarge and rehabilitate the old masonry building constituting the Liceo Ginnasio Napoleonico since 1833 and, before, the Convent of Santa Elisabetta. Part of the new building is founded on the old convent, while the other part rests on the ground. The building has an L-shaped plan formed by two wings, indicated in Fig. 1a and Fig. 1b with "A" and "B". The structure consists of reinforced concrete frames; beams have a rectangular section, excepting those on the perimeter, which are characterized by cross-sections with a tapered articulated geometry (i.e. with variable height and width) (Fig. 1c). All columns have a square cross-section rotated by $45^{\circ}$ with respect to the direction of the frames for architectural reasons, with dimensions equal to $35 \times 35 \mathrm{~cm}$, excepting two inner columns of body "A" that have a $42 \times 42 \mathrm{~cm}$ cross-section.

In 2013 the building was seismically retrofitted through the construction of two Dissipative Towers [8] (Fig. 1d), one connected both to body "A" and to body "B" (Tower A) and the other only to body "B" (Tower B). The towers rest on a thick reinforced concrete plate connected by a central spherical hinge to a second slab founded on piles. Simplifying as much as possible the description of the retrofit, it can be stated that the Towers have the twofold aim (i) to regularize the horizontal displacements of the building, favoring a linear deflection (i.e. constant interstory drifts), and (ii) to increase the dissipation of the seismic input energy through viscous 
dissipative devices that exploit the relative velocities between the two reinforced concrete plates at the base of each Tower.

During the seismic retrofit, dynamic ambient vibration tests were performed to identify the modal parameters of the building. A first dynamic identification was conducted in 2012, before the intervention, and a second one was carried out in 2013 upon completion of the upgrading work. The purpose of the investigations carried out was twofold: on one hand, the preliminary identification tests allowed to obtain information on the global dynamic behavior of the building to support the design of the intervention and the calibration of a finite element model; on the other hand, the investigations in the post-operam situation allowed to verify that the modal parameters of the retrofitted structure matched those predicted by the numerical model. More details on the intervention, which is heavily constrained by technical requirements, can be found in Balducci et al. (2015) [9], while a more extensive description of the tests and the relevant results can be found in Gara et al. (2021) [10].
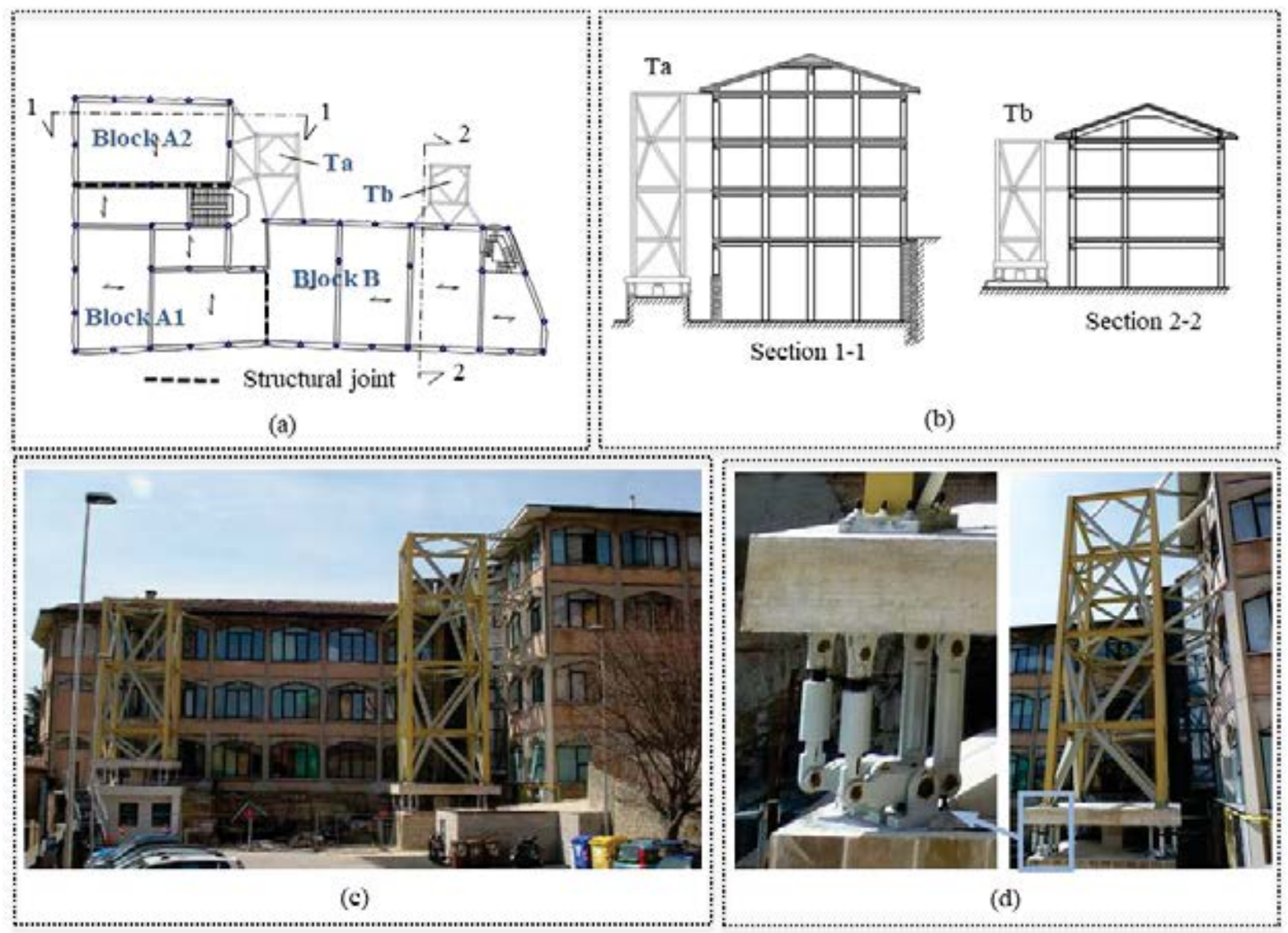

Figure 1: The Costanza da Varano high school: (a) plan view; (b) cross-section; (c) photo of the building; (d) photo of the Dissipative Towers with details of the dampers.

\section{DESCRIPTION OF THE DYNAMIC MONITORING}

After the seismic event of magnitude 6.0 occurred on August 24, 2016, a new ambient vibration test was performed on August 27, 2016, with the purpose of verifying the health of the structure. Fig. 2a shows the sensor configuration adopted for each floor of the building while Fig. $2 \mathrm{~b}$ shows a photo of the acquisition system. As for the instrumentation, PCB 393B31 piezoelectric sensors with a sensitivity of $10 \mathrm{~V} / \mathrm{g}$, NI 9234 analog-to-digital conversion boards with a resolution of 24 bits, a cDAQ 9178 chassis, and a notebook for data acquisition and 
storage have been used. A sampling rate of $2048 \mathrm{~Hz}$ has been used for the acquisitions. Fig. 2c shows the frequency content of the recordings carried out while Fig. $2 \mathrm{~d}$ and Fig. $2 \mathrm{e}$ show the results of the dynamic identification performed using the SSI-COV algorithm and the MAC (Modal Assurance Criterion) matrix. It is worth to specify that the building dynamics after the event of August 2016 remained unchanged from that identified after the retrofitting in 2013.

Following this test, a continuous dynamic monitoring system has been installed on the structure for three days; the system was composed by the same instrumentation adopted to perform the benchmark tests but additional accelerometers were installed at the foundation level in order to record the seismic input. Ambient vibration tests at the beginning of the monitoring allowed the identification of modal parameters that were subsequently traced via the continuous monitoring system. During the monitoring days (from August 27 to 29, 2017) several earthquakes occurred; the event with the highest intensity of magnitude 4.2 was registered on August 28 and occurred approximately $37 \mathrm{~km}$ away from the building.

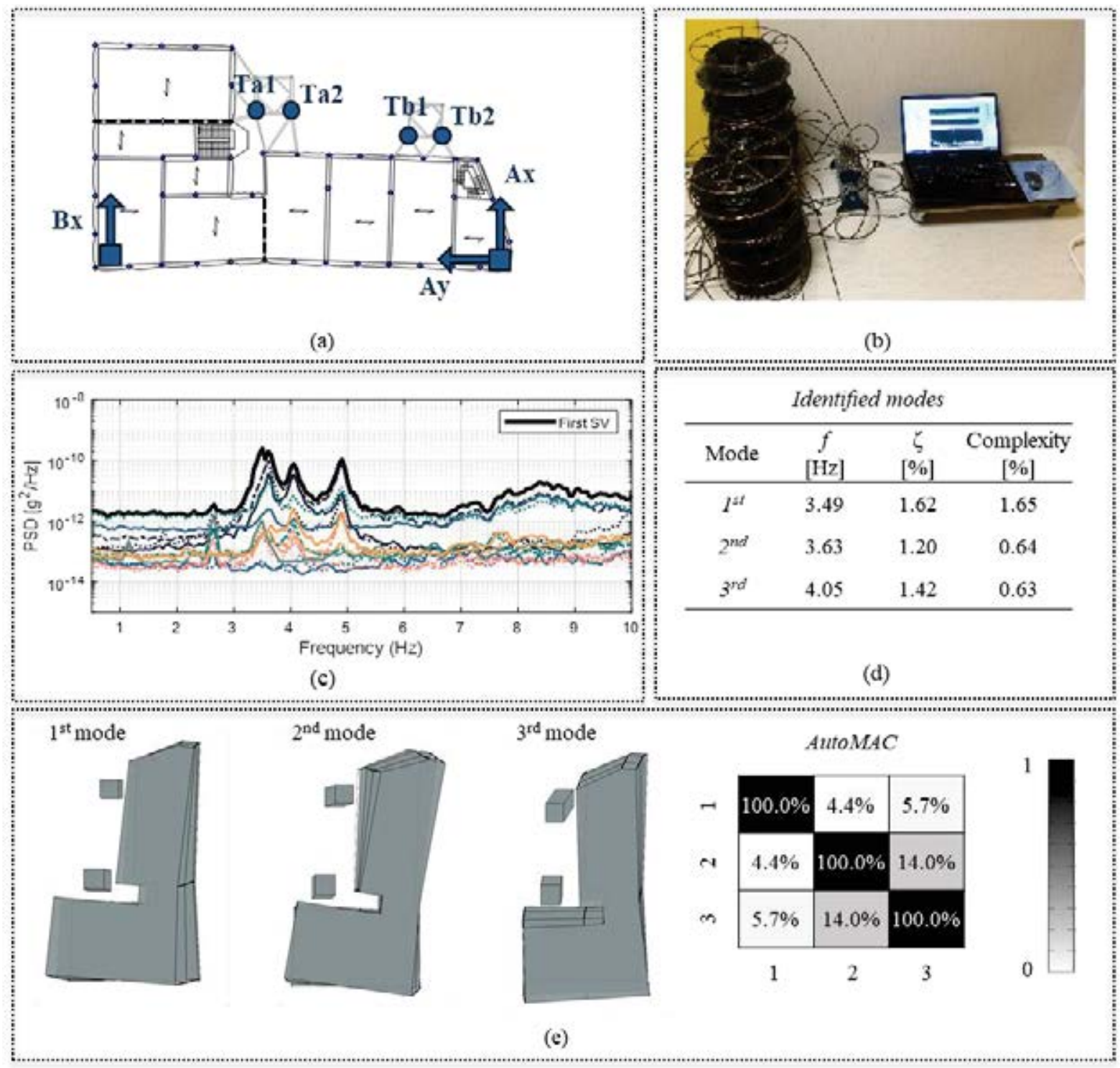

Figure 2: a) Sensors configuration adopted for each floor; b) acquisition system; c) frequency content from measurements during ambient vibration test; d) results of the dynamic identification; e) identified modal shapes and relevant AutoMAC matrix. 


\section{DESCRIPTION OF THE LINEARIZATION PROCEDURE}

For all the registered events, the structure exhibited a nonlinear response that made it difficult to identify its modal parameters from the seismic response. Fig. 3 shows a time-frequency analysis, performed using the Short Time Fourier Transform (STFT), performed on the measurement of the magnitude 4.2 event for the sensor on the top floor (location Ay). From Fig. 3, it is possible to observe the non-stationarity of the structural response, which leads the need of implementing a method to linearize the structural response.

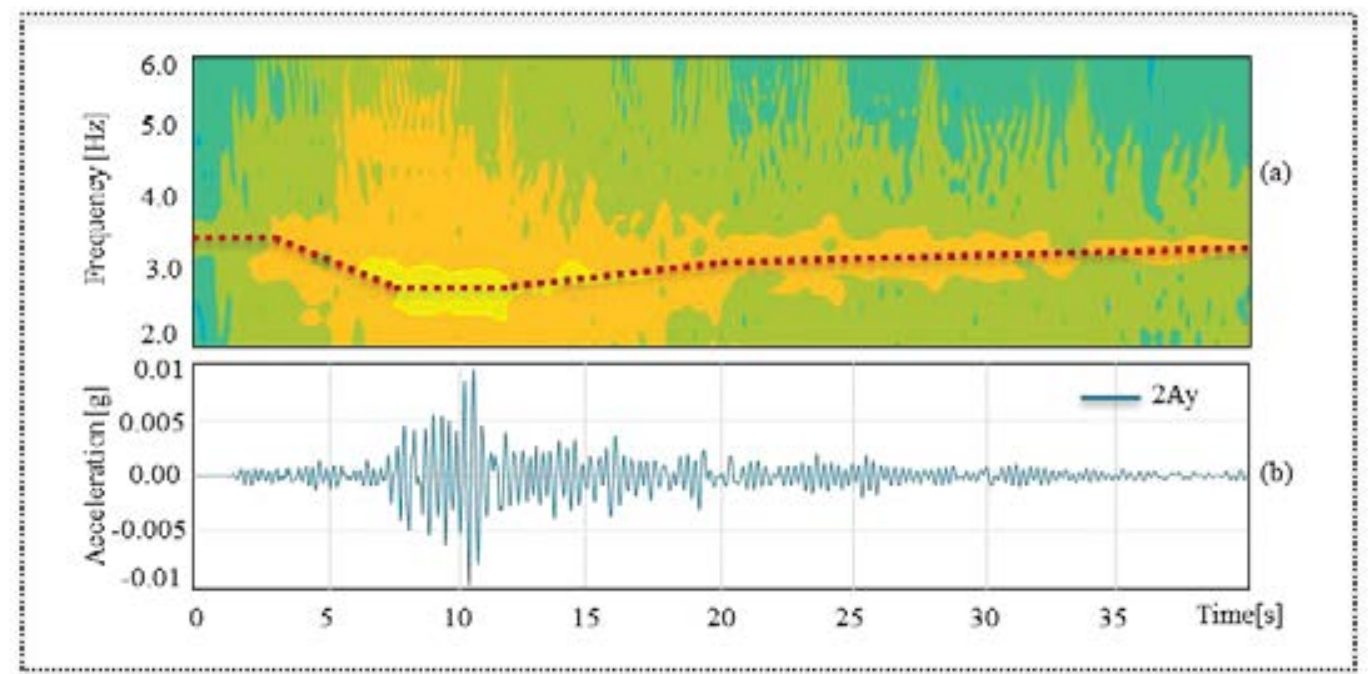

Figure 3: Short Time Fourier Transform carried out on a record made during the magnitude 4.2 event.

The proposed algorithm takes advantage of the subspace identification methods; the latter are well established in the civil engineering field and are considered as robust and reliable algorithms. The recurrent algorithms in the literature, for the identification of MIMO systems are the Multivariable Output Error State Space (MOESP) [11] and the numerical algorithm for Subspace State Space System IDentification (N4SID) [12]. Many works can be found in which above algorithms have been successfully used for dynamic identification of full-scale case studies during seismic events $[13,14,15,16]$. The first step in using the subspace identification algorithms is to write the dynamical system in the state space, through a state equation (Eq. 1) and an output equation (Eq. 2):

$$
\begin{gathered}
\mathbf{x}_{k+1}=\mathbf{A} \mathbf{x}_{k}+\mathbf{B} \mathbf{u}_{k}+\mathbf{w}_{k} \\
\mathbf{y}_{k}=\mathbf{C} \mathbf{x}_{k}+\mathbf{D} \mathbf{u}_{k}+\mathbf{v}_{k}
\end{gathered}
$$

where $\mathbf{u}_{k} \in \mathbb{R}^{m}$ and $\mathbf{y}_{k} \in \mathbb{R}^{l}$ denote the input and output signals, respectively, at a certain time $k$, while $\mathbf{x}_{k} \in \mathbb{R}^{n}$ is the state vector. In addition, $\mathbf{A} \in \mathbb{R}^{n \times n}$ is the dynamical system matrix, $\mathbf{B} \in$ $\mathbb{R}^{n \times m}$ is the input matrix that describes how the deterministic inputs influence the next state, $\mathbf{C} \in \mathbb{R}^{l \times n}$ is the output matrix that characterizes how the internal state influences the outputs and $\mathbf{D} \in \mathbb{R}^{l \times m}$ is the direct transition matrix. For a linear time-invariant system above matrices are constant. Furthermore, $\mathbf{w}_{k} \in \mathbb{R}^{n}$ and $\mathbf{v}_{k} \in \mathbb{R}^{l}$ are unmeasurable vector signals, which are assumed to be normally distributed, zero mean, white noise signals.

For the case study under investigation, the structural response to seismic events of the building at hand is clearly time-varying, and it can therefore be assumed that the system matrices of the state space model change with time $k$. Several works in the literature deal with methods for 
the identification of time-varying systems; in particular, some interesting algorithms are the MOESP-VAR [17], the N4SID-VAR [18], and the one proposed in Loh and Chen [19].

In this paper, an iterative procedure for the identification of the time-varying dynamical system is proposed, based on the optimization of the number of samples, and thus the length of the signal windows, in which the dynamics of the system can be described as a linear time-invariant process. The length of the first window is selected from the results of the time-frequency analysis and varied until the identified dynamic model accurately reproduces the experimental response starting from the measured input. The steps of the optimization procedure are summarized in the flowchart of Fig. 4; the identification within each window was done through the "robust combined algorithm" proposed by Van Overschee and De Moor in [12]. The accuracy of the identified model in reproducing the building response is evaluated comparing the predicted and measured time histories of accelerations through the comparison metrics proposed by Kavrakov et al. [20], which consider several signal properties such as phase, peak, root mean square, and frequency contents. A detailed explanation of the approach can be found in Gara et al. (2021) [21].

Fig. 5 shows the results obtained by applying the proposed procedure on the seismic response of the building to the magnitude 4.4 event. In particular, Fig. 5a shows the comparison between the signal recorded at the measurement point Ay at the top floor and the response estimated by the identified time-varying dynamical system. Figs. $5 \mathrm{~b}$ and $5 \mathrm{c}$ show the results in terms of modal parameters (resonant frequencies and damping ratios) and reveal how the first three frequencies of the structure decrease during the strong motion and then gradually come back to the initial values at the end of the event; an opposite trend is observed for the damping ratios. The explanation is probably to be sought in the nonlinear phenomena, e.g. light infill cracking, frictions, and interaction phenomena between structural and non-structural members that are triggered above a certain level of excitation provided to the structure.

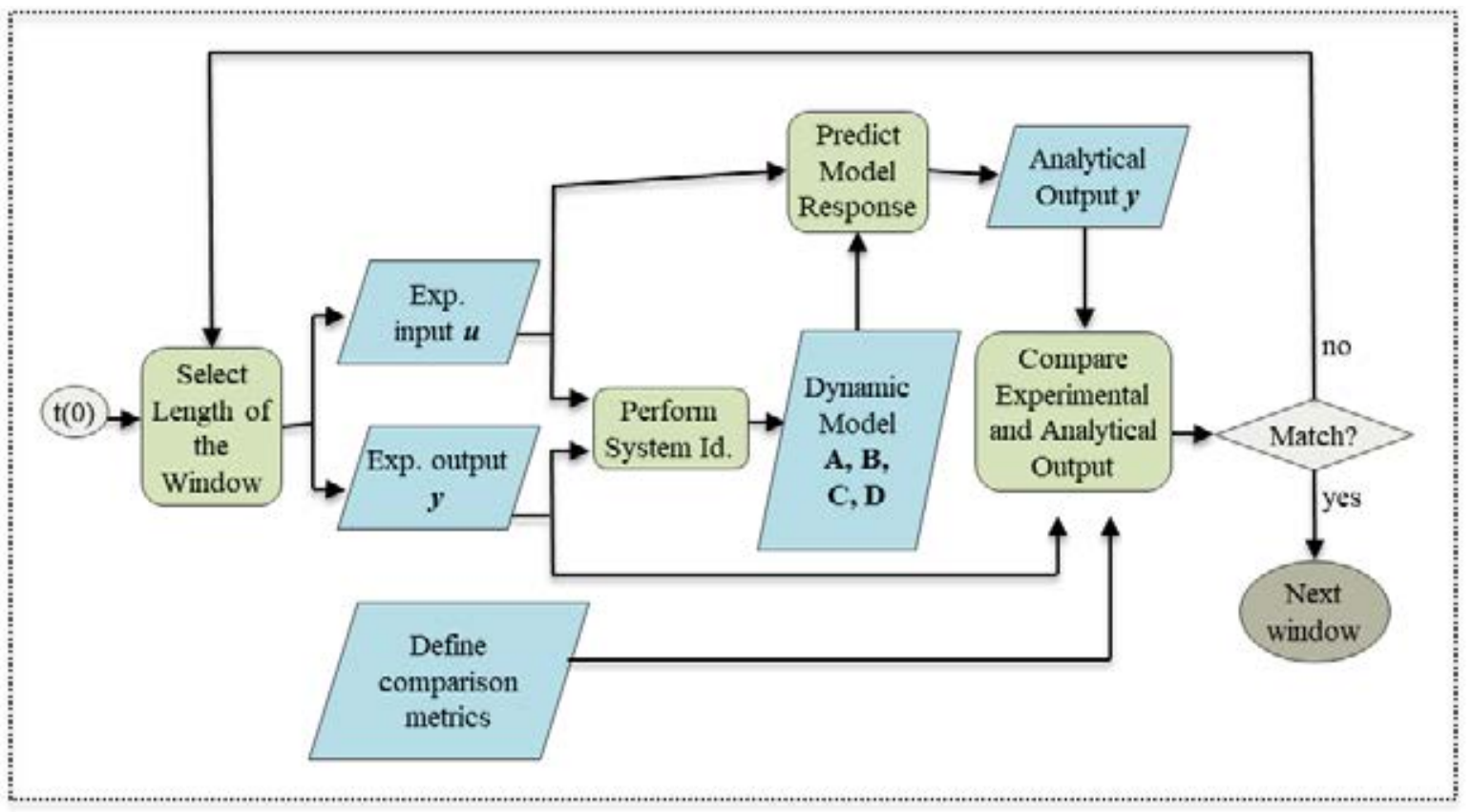

Figure 4: Flow chart of the proposed identification algorithm. 


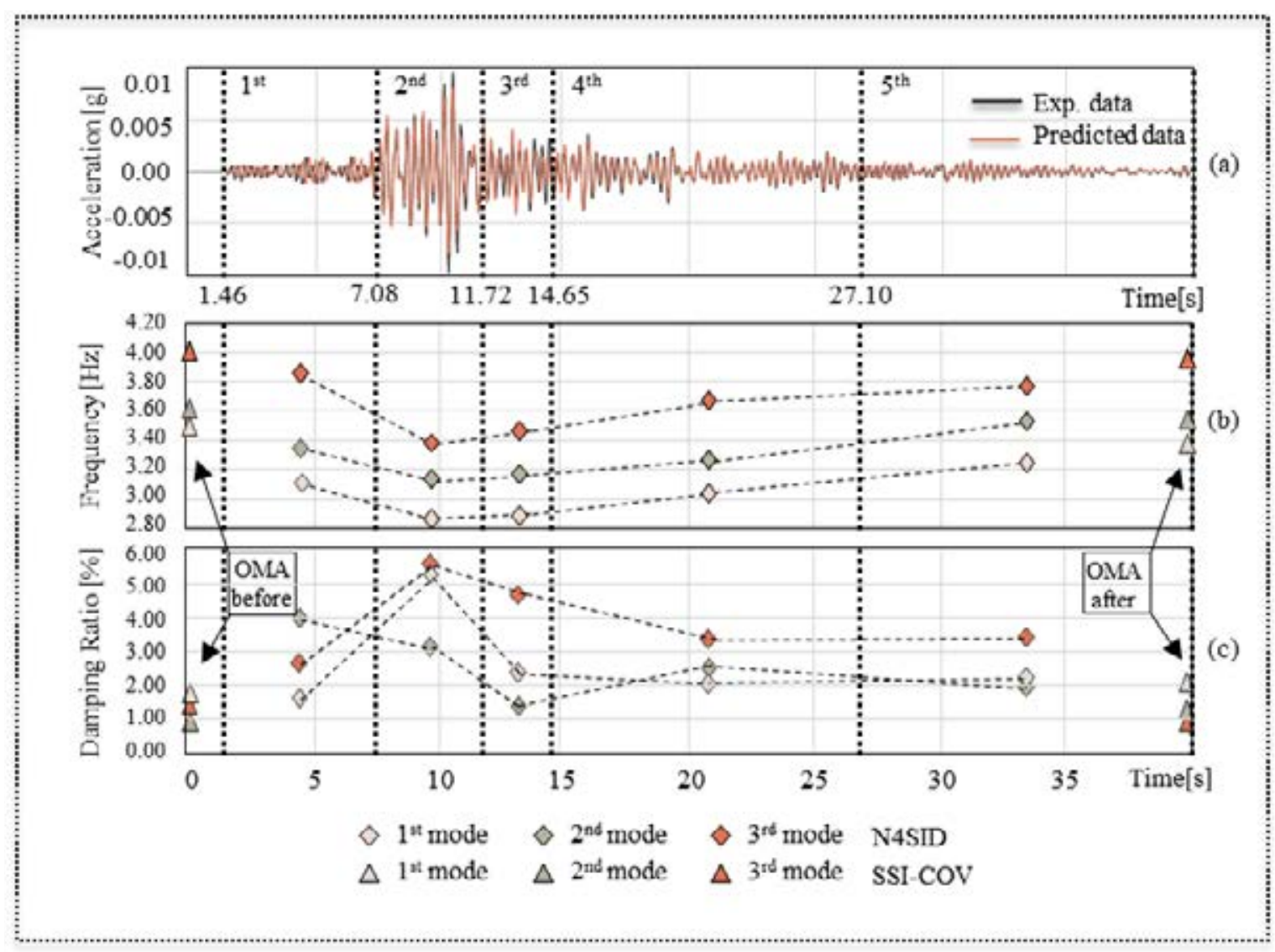

Figure 5: Laboratory mock-up case study.

\section{CONCLUSIONS}

An approach for tracking the fundamental frequencies of monitored buildings accounting for their nonlinear response triggered by low-medium intensity earthquakes has been presented in this paper. The procedure, consisting of an iterative algorithm aimed at identifying signal windows in which the dynamics of the system can be described as a linear time-invariant process, is applied to a record extracted from the monitoring of a school building located in Camerino during the seismic swarm that followed the August 2016 Central Italy earthquake. The results highlighted the nonlinearity of the building seismic response even in the absence of damage, providing a useful tool for the interpretation of accelerometric data in structural health monitoring.

\section{REFERENCES}

[1] Clinton, J. F., Bradford, S. C., Heaton, H. T., and Favela, J. (2006). "The Observed Wander of the Natural Frequencies in a Structure." Bulletin of the Seismological Society of America 96 (1): 237-57. https://doi.org/10.1785/0120050052.

[2] Regni, M., Arezzo, D., Carbonari, S., Gara, F., and Zonta, D. (2018). "Effect of Environmental Conditions on the Modal Response of a 10-Story Reinforced Concrete Tower." Shock and Vibration 2018 (July): 1-16. https://doi.org/10.1155/2018/9476146. 
[3] Rainieri, C., Magalhaes, F., Gargaro, D., Fabbrocino, G., and Cunha, A. (2018). "Predicting the Variability of Natural Frequencies and Its Causes by Second-Order Blind Identification." Structural Health Monitoring 18 (2): 486-507. https://doi.org/10.1177/1475921718758629.

[4] Rainieri, C., Gargaro, D., and Fabbrocino, G. (2019). "Hardware and Software Solutions for Seismic SHM of Hospitals." In Seismic Structural Health Monitoring, edited by Maria Pina Limongelli and Mehmet Çelebi, 279-300. Springer Tracts in Civil Engineering. Cham: Springer International Publishing. https://doi.org/10.1007/978-3-030-13976-6_12.

[5] Gentile, C., Antonello, R., and Saisi, A. (2019). "Continuous Dynamic Monitoring to Enhance the Knowledge of a Historic Bell-Tower." International Journal of Architectural Heritage 13 (7): 992-1004. https://doi.org/10.1080/15583058.2019.1605552.

[6] Cavalagli, N., Comanducci, G., and Ubertini, F. (2018). "Earthquake-Induced Damage Detection in a Monumental Masonry Bell-Tower Using Long-Term Dynamic Monitoring Data." Journal of Earthquake Engineering 22 (sup1): 96-119. https://doi.org/10.1080/13632469.2017.1323048.

[7] Ubertini, F., Cavalagli, N., Kita, A., and Comanducci, G. (2018). “Assessment of a Monumental Masonry Bell-Tower after 2016 Central Italy Seismic Sequence by Long-Term SHM." Bulletin of Earthquake Engineering $16 \quad$ (2): $775-801$. https://doi.org/10.1007/s10518-017-0222-7.

[8] Balducci, A., 2005. "Dissipative Towers". Application n. EP20100747238, PCT n. WO2010EP62748 20100831, International and European classification E04H9/02 - Italian concession $\mathrm{n}^{\circ} 0001395591$.

[9] Balducci, A., and Castellano, M. G. (2015). "Adeguamento sismico del liceo Varano di Camerino mediante sistema a torri dissipative." Progettazione Sismica, no. 01: 69-91. https://doi.org/10.7414/PS.6.1.69-91.

[10] Gara, F., Carbonari, S., Roia, D., Balducci, A., and Dezi L. (2021). "Seismic retrofit assessment of a school building through operational modal analysis and f.e. modelling”. J. Struct. Eng. (ASCE). doi:10.1061/(ASCE)ST.1943-541X.0002865.

[11] Verhaegen, M. (1994). "Identification of the Deterministic Part of MIMO State Space Models given in Innovations Form from Input-Output Data." Automatica 30 (1): 61-74. https://doi.org/10.1016/0005-1098(94)90229-1.

[12] Van Overschee, P., and De Moor, B. (1996). Subspace Identification for Linear Systems. Boston, MA: Springer US. https://doi.org/10.1007/978-1-4613-0465-4.

[13] Ceravolo, R., Matta, E., Quattrone, A., and Zanotti Fragonara, L. (2017). “Amplitude Dependence of Equivalent Modal Parameters in Monitored Buildings during Earthquake Swarms: Amplitude Dependence of Modal Parameters in Monitored Structure." Earthquake Engineering \& Structural Dynamics $46 \quad$ (14): 2399-2417. https://doi.org/10.1002/eqe.2910

[14] Skolnik, D., Lei, Y. Yu, E., and Wallace., J. W. (2006). "Identification, Model Updating, and Response Prediction of an Instrumented 15-Story Steel-Frame Building." Earthquake Spectra 22 (3): 781-802. https://doi.org/10.1193/1.2219487.

[15] García-Illescas, M. Á., Murià-Vila, D., and Alvarez-Icaza, L. (2019). "Monitoring and Identification of Vibration Frequencies on a Portion of México City Metro Line 12." Advances in Civil Engineering 2019 (July): 1-13. https://doi.org/10.1155/2019/4128320. 
[16] Boroschek, R. L. (2013). "Structural Health Monitoring Performance During the 2010 Gigantic Chile Earthquake." In Earthquakes and Health Monitoring of Civil Structures, edited by Mihail Garevski, 197-216. Springer Environmental Science and Engineering. Dordrecht: Springer Netherlands. https://doi.org/10.1007/978-94-007-5182-8_8.

[17] Tamariz, A.D.R., Bottura, C.P., and Barreto, G. (2005). "Iterative MOESP Type Algorithm for Discrete Time Variant System Identification." In Proceedings of the 2005 IEEE International Symposium on, Mediterrean Conference on Control and Automation Intelligent Control, 2005., 399-404. Limassol, Cyprus: IEEE. https://doi.org/10.1109/.2005.1467048.

[18] Robles, A. E., and Giesbrecht, M. (2018). "N4SID-VAR Method for Multivariable Discrete Linear Time-Variant System Identification." In Proceedings of the 15th International Conference on Informatics in Control, Automation and Robotics, 502-9. Porto, Portugal: SCITEPRESS - Science and Technology Publications. https://doi.org/10.5220/0006907505020509.

[19] Loh, C., and Chen, J. (2017). "Tracking Modal Parameters from Building Seismic Response Data Using Recursive Subspace Identification Algorithm: Tracking Modal Parameters Using Recursive Subspace Identification.” Earthquake Engineering \& Structural Dynamics 46 (13): 2163-83. https://doi.org/10.1002/eqe.2900.

[20] Kavrakov, I., Ahsan K., and Morgenthal, G. (2020). "Comparison Metrics for Time-Histories: Application to Bridge Aerodynamics.” Journal of Engineering Mechanics 146 (9): 04020093. https://doi.org/10.1061/(ASCE)EM.1943-7889.0001811.

[21] Gara F., Arezzo D., Nicoletti V., Carbonari S. "Monitoring of the Modal Properties of a RC School Building During the Central Italy Seismic Swarm." Journal of Structural Engineering (ASCE) 147(7):05021002. https://doi.org/10.1061/(ASCE)ST.1943541X.0003025. 